\title{
INVESTIGATION OF MIXING TIME IN LIQUID UNDER INFLUENCE OF ROTATING MAGNETIC FIELD
}

\author{
Alicja Przybył ${ }^{*}$, Rafał Rakoczy ${ }^{1}$, Maciej Konopacki ${ }^{1}$, Marian Kordas ${ }^{1}$, \\ Radosław Drozd ${ }^{2}$, Karol Fijałkowski ${ }^{2}$ \\ ${ }^{1}$ West Pomeranian University of Technology Szczecin, Faculty of Chemical Technology and \\ Engineering, Institute of Chemical Engineering and Environmental Protection Processes, al. Piastów \\ 42, 71-065 Szczecin, Poland \\ ${ }^{2}$ West Pomeranian University of Technology Szczecin, Faculty of Biotechnology and Animal \\ Husbandry, Department of Immunology, Microbiology and Physiological Chemistry, al. Piastów 45, \\ 70-311 Szczecin, Poland
}

\begin{abstract}
The aim of the study was to present an experimental investigation of the influence of the RMF on mixing time. The obtained results suggest that the homogenization time for the tested experimental set-up depending on the frequency of the RMF can be worked out by means of the relationship between the dimensionless mixing time number and the Reynolds number. It was shown that the magnetic field can be applied successfully to mixing liquids.
\end{abstract}

Keywords: rotating magnetic field, mixing time, hydrodynamics, mixing effect

\section{INTRODUCTION}

The mixing process is very often applied in chemical engineering systems and is the subject of many experimental investigations. This process is often realised by means of the mixing tank or stirred vessel and it may be described by various parameters (Zlotarnik, 2001).

The mixing time and power consumption of the agitator or mixing time are important parameters for a mixer or reactor design (Harnby et al., 2000; Stręk, 1981). Power consumption is a basic quantity in a mixing process that, in part, determines other process quantities, e.g. mixing time. It should be also noticed that the cost of a process depends on the power consumption (King et al., 1988). In many situations, the correct knowledge of the power consumption and the mixing time are needed for better design and operation of the various types of mixers (Hiraoka et al., 2001).

The mixing time can be compared with mass transfer time or reaction time when evaluation of the controlling mechanism of the process is of interest (Bouaifi and Rousatn, 2001). This parameter represents one the most useful criteria for characterization of the mixing system design, scaling-up and optimization of the mixing process (Jaworski et al., 2000; Karcz et al., 2005). This parameter contains information about hydrodynamics and mixing within the mixer and can be useful for the scaling up (Cascaval et al., 2004). The design process of a novel type of mixer closely depends on knowledge of hydrodynamics in the mixing vessel. Therefore, the mixing time is often used as an indication of the effectiveness of a mixing system (Kordas et al., 2013). The mixing time denotes the time required for the tank composition to achieve a specified level of homogeneity following the addition of a tracer pulse at a single point in a mixer (Hadjiev et al., 2006). This parameter is also defined as the time 
needed to reach a given mixing intensity when starting from the completely segregated situation (Oniscu et al., 2002).

The mixing performance of stirred tanks equipped with various types of agitators has been analyzed by many researchers (Masiuk and Rakoczy, 2007; Masiuk et al., 2008). Most investigations have examined the mixing time and power consumption of agitation systems resulting in empirical correlations (Bouaifi and Roustan, 2001; Manjula et al. 2009; Szoplik and Karcz, 2008; Woziwodzki et al., 2010; Zadghaffari et al., 2006).

A novel approach to the mixing process focuses on the application of a rotating magnetic field (RMF). This kind of magnetic field may be used to augment the process intensity instead of mechanical intensity (Rakoczy, 2013). It should be noticed that RMF may act as a non-instructive mixing device (Moffat, 1965; Moffat, 1991).

The main motivation for the present work was to investigate the possibility of the mixing system provided with a generator of RMF for the mixing process of various types of liquids, including water, $\mathrm{NaCl}$ solutions, Hestrin-Schramm medium (as an example of the microbiological medium used in the biotechnological process of bacterial cellulose production). The interaction of the applied magnetic field (MF) with liquids can be described by the relation between the mixing time and the Reynolds numbers. These dimensionless numbers allow for quantitative representation and characterization of the influence of the hydrodynamic condition under the RMF action on the mixing process.

\section{THEORETICAL BACKGROUND}

The mixing time, defined as the time needed to reach a specific degree of homogeneity, is defined by means of a dimensionless mixing time defined by the formula (Rakoczy, 2013):

$$
\Theta=f\left(\operatorname{Re}_{R M F}\right)
$$

The above relation includes a non-dimensional group characterizing the mixing time problem, expressed in the formula (Kordas, 2013):

$$
\Theta=\frac{t_{95} v}{D^{2}}
$$

To characterize the mixing process under the action of the magnetic field, the Reynolds number based on the frequency of the RMF may be used in the following form (Story et al., 2016):

$$
\operatorname{Re}_{R M F}=\frac{\Omega_{R M F} D^{2}}{v}
$$

The parameter $\Omega_{R M F}$ may be expressed as follows (Moffat, 1965):

$$
\Omega_{R M F}=\frac{\left.w_{\varphi}\right|_{\max }}{D}
$$

The maximum peripheral speed of the mixed liquid under the action of the RMF may be expressed as (Dahlberg, 1972):

$$
\left.w_{\varphi}\right|_{\max }=B_{\max } D \sqrt{\frac{\omega_{R M F} \sigma_{e}}{\rho}}
$$




\section{MATERIALS AND METHODS}

\subsection{Experimental apparatus}

Investigations were performed using the experimental apparatus shown in Fig. 1. The experimental apparatus consisted of a cooling jacket (1) and a generator of RMF (2). The RMF was generated by the 3 -phase stator of the squirrel cage induction motor. The power of the stator winding used 3-phase alternating current. The container (3) was axially aligned with the RMF generator. The mixing system consisted of a vessel of $145 \mathrm{~mm}$ inner diameter and $415 \mathrm{~mm}$ height. The a.c. transistorized inverter (4) was used to adjust the RMF frequency, $f$, in the range of $10-50 \mathrm{~Hz}$, and to regulate the maximum voltage in the range of 10-100 V. This inverter was connected with a personal computer (5) equipped with software to control the RMF generator. Additionally, recorders (6) with electrodes (7) were used to measure the mixing time.

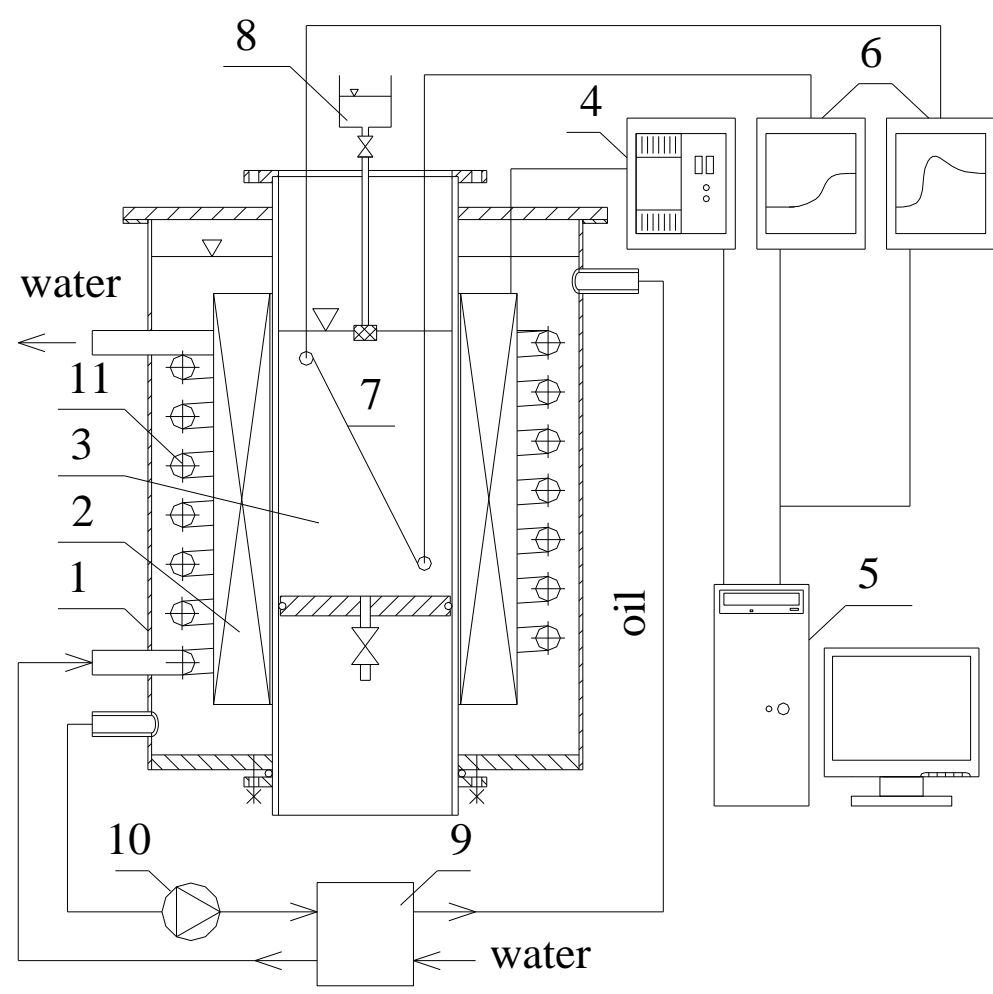

Fig. 1. Scheme of experimental apparatus: 1 - cooling jacket, 2 - RMF generator, 3 - vessel, 4 - a.c. transistorized inverter, 5 - personal computer, 6 - recorders (CX-701), 7 - electrodes, 8 - batcher, 9 - heat exchanger, 10 pump, 11 - internal coil

The tracer, (sodium hydroxide solution; $1 \mathrm{~mol} \cdot \mathrm{dm}^{-3}$ ) was introduced into the working liquid using the batcher (8). The temperature of the analyzed liquids during the exposition to the RMF was controlled by an additional cooling system based on oil circulation (in a heat exchanger (9) and a pump (10) and water circulation system in an internal coil (11).

\subsubsection{Working liquids}

A total of $5 \mathrm{dm}^{3}$ liquid was introduced into the glass container. Aqueous solutions of $\mathrm{NaCl}$ brine, distilled water and Herstin-Schramm (HS) medium composed of glucose $(2 \mathrm{w} / \mathrm{v} \%)$, yeast extract $(0.5$ w/v\%), bacto-pepton $(0.5 \mathrm{w} / \mathrm{v} \%)$, citric acid $\left(0.115 \mathrm{w} / \mathrm{v} \%\right.$, ) $\mathrm{Na}_{2} \mathrm{HPO}_{4}(0.27 \mathrm{w} / \mathrm{v} \%), \mathrm{MgSO}_{4} \cdot 7 \mathrm{H}_{2} \mathrm{O}(0.05$ $\mathrm{w} / \mathrm{v} \%)$ and ethanol (1 v/v\%) (Ciechańska et al., 1998). The HS medium is an example of the 
microbiological growth medium used in the biotechnological process of bacterial cellulose production (Fijałkowski et al., 2015). The temperature of the analyzed liquids was equal to $20^{\circ} \mathrm{C}$. This parameter was monitored using the special temperature sensors cooperating with the recorder.

The physical parameters of the applied fluids are given in Table 1.

Table 1. Physical properties of tested liquids (for temperature $T=20^{\circ} \mathrm{C}$ )

\begin{tabular}{|l|c|c|c|}
\hline \multicolumn{1}{|c|}{ Medium } & $\rho, \mathrm{kg} \cdot \mathrm{m}^{-3}$ & $\eta, \mathrm{Pa} \cdot \mathrm{s}$ & $\sigma_{\mathrm{e}}, \mathrm{A}^{2} \cdot \mathrm{s}^{3} \cdot \mathrm{kg}^{-1} \cdot \mathrm{m}^{-3}$ \\
\hline distilled water & 998 & 0.001002 & 0.05 \\
\hline $4 \% \mathrm{w} / \mathrm{w} \mathrm{NaCl}$ brine & 1027 & 0.001068 & 6.35 \\
\hline $8 \% \mathrm{w} / \mathrm{w} \mathrm{NaCl}$ brine & 1056 & 0.001145 & 14.00 \\
\hline Hestrin-Schramm (HS) medium $\left.{ }^{*}\right)$ & 986 & 0.000944 & 0.65 \\
\hline
\end{tabular}

${ }^{*}$ Viscosity of H-S medium was obtained using a capillary viscometer

\subsubsection{Mixing time measurements}

The mixing time was determined using the $\mathrm{pH}$ tracer method (Kushalkar and Pangarkar, 1994). The values of this parameter were determined by means of top injections of $1 \mathrm{M} \mathrm{NaOH}$ used as a tracer. The top injection means that the tracer is added at the top of the vessel, $1 \mathrm{~cm}$ under the liquid surface and in the middle of the tested apparatus. All injections were performed with a batcher. The time injection was equal to $6 \mathrm{~s}$.

Two $\mathrm{pH}$ electrodes were used as follows: one probe mounted at $10 \mathrm{~mm}$ from the vessel bottom; another probe was mounted opposite the bottom $\mathrm{pH}$ electrode. Both were connected to the recorder (CX-701). The localization of the electrodes and the injection point are shown in Fig. 2.

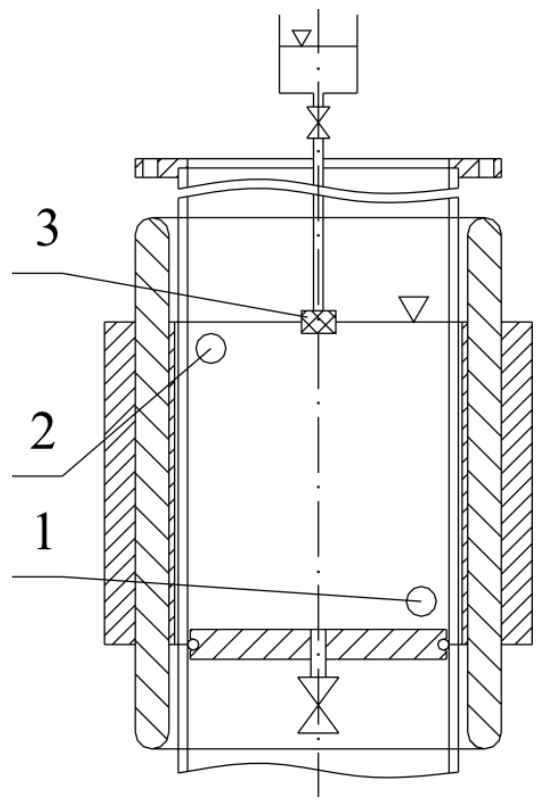

Fig. 2. Localization of the $\mathrm{pH}$ electrodes $(1,2)$ and the injection point $(3)$

As a tracer, $50 \mathrm{ml}$ of sodium hydroxide solution $\left(1 \mathrm{~mol} \cdot \mathrm{dm}^{-3}\right)$ was injected. The $\mathrm{pH}$ tracer method was based on measurements of $\mathrm{pH}$ difference response caused by the tracer $(1 \mathrm{M} \mathrm{NaOH})$. The mixing process was completed when the $\mathrm{pH}$ of the mixed liquid stopped changing. The time to reach, $t_{95}$, (95\% of the concentration step change from starting point to end point concentration) was defined as the 
mixing time, that is, the time necessary to render the liquid well-mixed. This parameter is defined as a time required for the system to mix the liquid to a prescribed final state and can be represented by the following relationship:

$$
Y=\frac{\left|p H(t)-p H_{0}\right|}{\left|p H_{\infty}-p H_{0}\right|}
$$

where $p H_{0}$ and $p H_{\infty}$ are the initial and the final $\mathrm{pH}$ values; $p H(\mathrm{t})$ is the value of $\mathrm{pH}$ at some instant in time $t$.

A typical example of the $\mathrm{pH}$ transient process is shown in Fig. 3. As shown in this figure, the mixing time $t_{95}$ for distilled water is much higher in comparison with the H-S medium.

a)

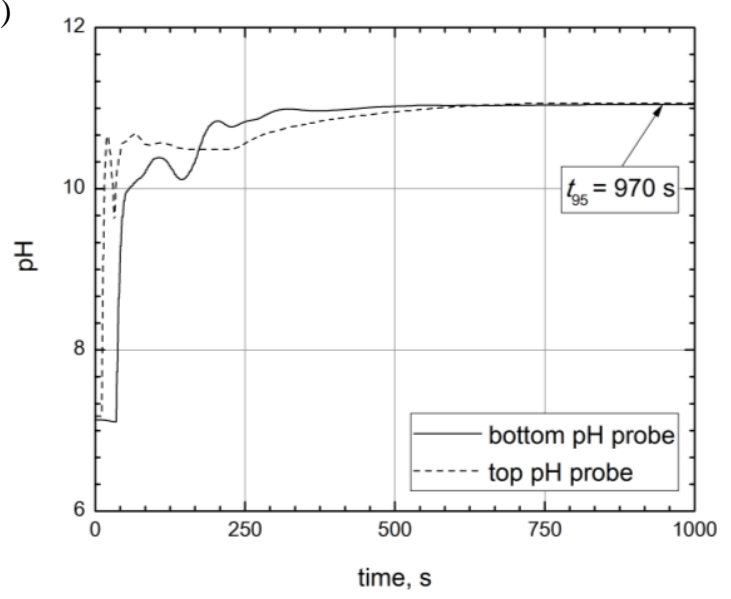

b)

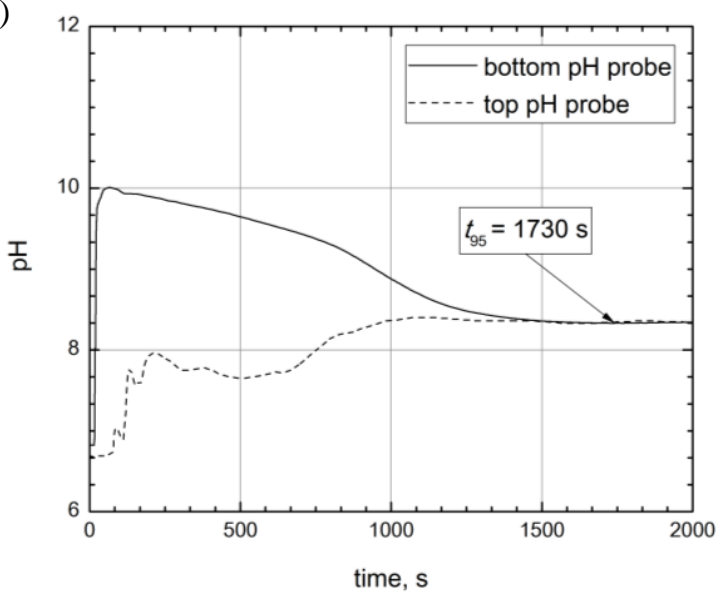

Fig. 3. Typical example of $\mathrm{pH}$ transient processes ( $\mathrm{pH}$ changes during mixing time investigation) at $f=30 \mathrm{~Hz}$ for:

a) H-S medium, b) distilled water

The mixing time is defined as the time required for the normalized probe output to reach and remain between 95 and $105 \%( \pm 5 \%)$ of the final equilibrium value. This value is called the $95 \%$ mixing time or $t_{95}$ (Magelli et al., 2013). To obtain an overall mixing time for the system, two probe responses must be combined and must be weighted toward the probe showing the largest concentration deviation to ensure that all regions of the vessel are mixed. This is achieved by means of the RMS variance (Paul et al., 2004).

\subsubsection{Magnetic field patterns}

The values of magnetic induction at different points inside the vessel are detected by means of microprocessor magnetic induction sensors connected with Hall probe. The measuring points were located on a plane that coincides with the axial section of the RMF generator. It should be noticed that the values of magnetic induction were recorded for about 110 points. Then, the averaged values of the magnetic field, $B_{a v g}$, were calculated as the arithmetic mean of these measurements.

As follows from the analysis of experimental data, the values of magnetic induction are spatially distributed in the volume of the RMF generator and may be presented in a system of coordinates $\left(R^{*}\right.$, $H^{*}$ ) in the form of iso-contour patterns (see Fig. 4). The normalized values of the radius, $R^{*}$, and the height, $H^{*}$, of a glass container, were defined as $R^{*}=R / R_{\max } \rightarrow R^{*}=R / 0.075 \mathrm{~m}$ and $H^{*}=H / H_{\max } \rightarrow H^{*}=H / 0.2 \mathrm{~m}$. 
a)

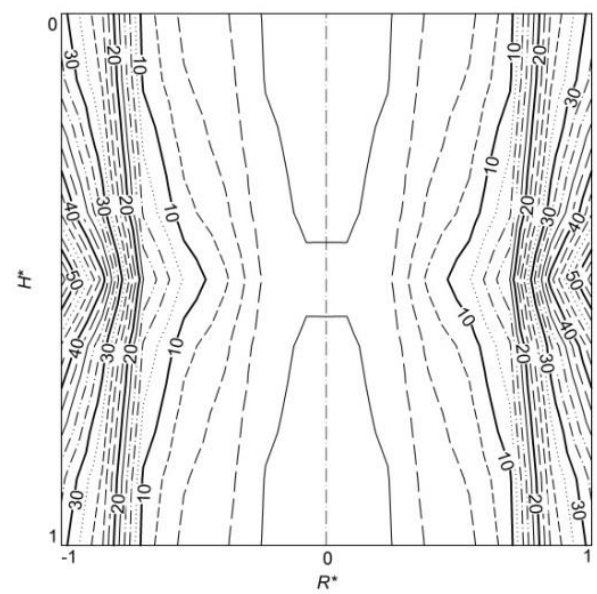

b)

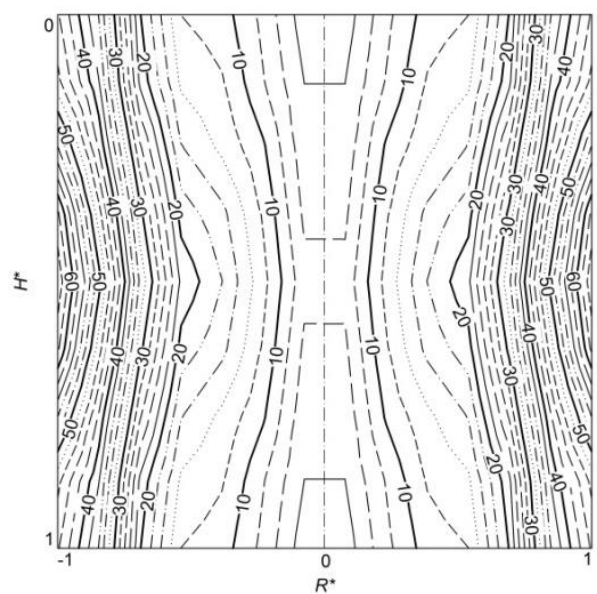

Fig. 4. A typical example of magnetic field patterns: a) $f=10 \mathrm{~Hz}$, b) $f=50 \mathrm{~Hz}$

As follows from the experimental database, the values of the magnetic induction, $B$, are spatially distributed. This distribution is depended on the RMF frequency, $f$. On the basis of the experimental measurements, the maximum values of magnetic induction, $B_{\max }$, were obtained. These values were recorded at the point $\left(R^{*}=-1\right.$ or $\left.1 ; H^{*}=0.5\right)$ inside the RMF generator. In order to establish the effect of the maximum and the averaged values of the magnetic field, the obtained results are graphically shown in Fig. 5.

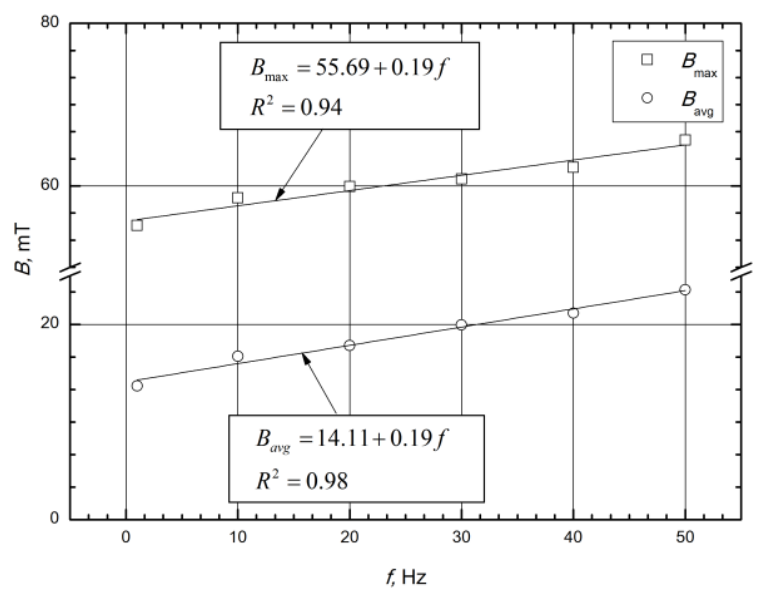

Fig. 5. Graphical presentation of relations between the maximum and averaged values of magnetic induction and RMF frequency

\section{RESULTS AND DISCUSSION}

Taking into account Eq. (1) and the basic parameters from Eqs (2) and (3), the following relationship was obtained:

$$
\begin{aligned}
& \Theta=f\left(\operatorname{Re}_{R M F}\right) \Rightarrow\left(\frac{t_{95} v}{D^{2}}\right)=f\left(\frac{\Omega_{R M F} D^{2}}{v}\right) \Rightarrow\left(\frac{t_{95} v}{D^{2}}\right)=f\left(\frac{\left.w_{\varphi}\right|_{\max }}{D} \frac{D^{2}}{v}\right) \\
& \Rightarrow\left(\frac{t_{95} v}{D^{2}}\right)=f\left(B_{\max } D \sqrt{\frac{\omega_{R M F} \sigma_{e}}{\rho}} \frac{D}{v}\right)
\end{aligned}
$$


According to Eq. (7), the plot of the data obtained in the current work was presented in coordinates $(\Theta$, $\left.\operatorname{Re}_{R M F}\right)$ of the log-log system in Fig. 6.

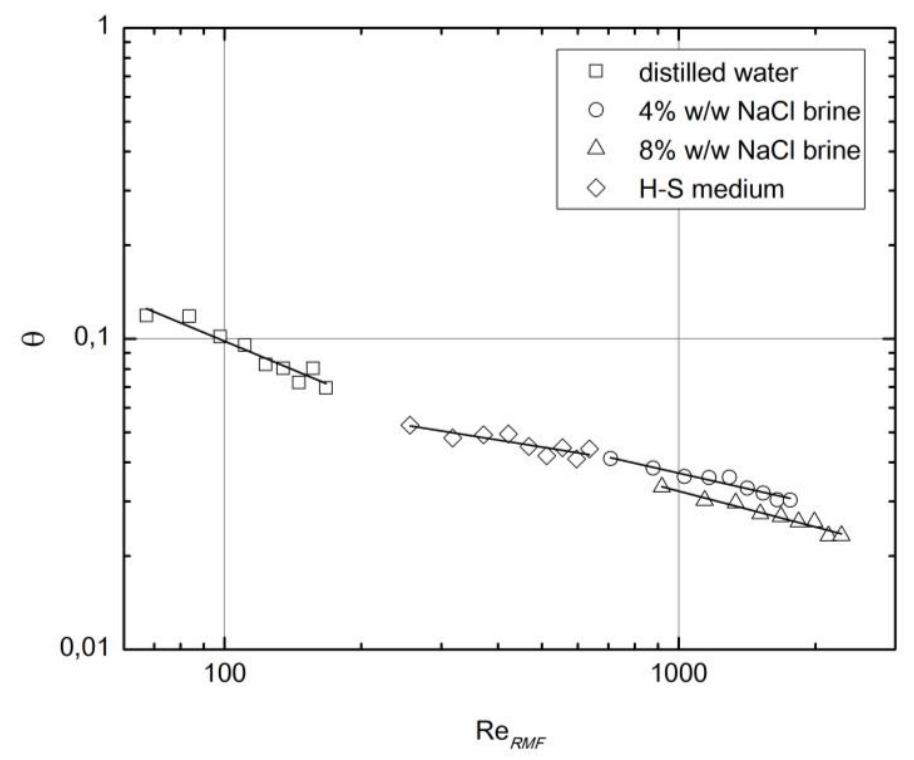

Fig. 6. The dependence of $\Theta=f\left(\operatorname{Re}_{R M F}\right)$ for the analyzed liquids

The experimental results presented in Fig. 6 indicate that the dimensionless mixing number versus the dimensionless Reynolds number may be defined by the following formula

$$
\Theta=p_{1}\left(\operatorname{Re}_{R M F}\right)^{p_{2}}
$$

The constants and exponents were computed by means of Matlab software and the principle of leastsquares. These values are collected in Table 2.

Table 2. Physical properties of tested liquids (for temperature $\mathrm{T}=20^{\circ} \mathrm{C}$ )

\begin{tabular}{|l|c|c|c|}
\hline \multicolumn{1}{|c|}{ Medium } & $p_{1}$ & $p_{2}$ & $R^{2}$ \\
\hline distilled water & 1.62 & -0.60 & 0.91 \\
\hline $4 \% \mathrm{w} / \mathrm{w} \mathrm{NaCl}$ brine & 0.36 & -0.33 & 0.94 \\
\hline $8 \% \mathrm{w} / \mathrm{w} \mathrm{NaCl}$ brine & 0.46 & -0.38 & 0.96 \\
\hline Hestrin-Schramm (HS) medium & 0.19 & -0.23 & 0.74 \\
\hline
\end{tabular}

According to Fig. 6, the scatter of experimental points for the various types of liquids may be described by the same relationship using various values of the coefficients (see Table 2). The results presented in Figure 6 show the influence of the dimensionless Reynolds number on the dimensionless mixing number. Figure 6 indicates also that the mixing time decreases moderately with the increase in the dimensionless Reynolds number. It was found that as the intensity of the magnetic field increased, the time duration of the mixing process under the action of the RMF decreased. Moreover, Fig. 6 shows a strong influence of the liquid on the analyzed mixing process. The dimensionless mixing time obtained for distilled water was consequently higher than that for the HS medium and $\mathrm{NaCl}$ solutions. Moreover, a significant decrease took place in the region of large values of the Reynold number. The obtained relationships (see Fig. 6 and data in Table 2) indicate that the mixing time is more sensitive to the dimensionless Reynolds number for distilled water $\left(\Theta \propto \operatorname{Re}^{-0.60}\right)$ than for HS medium $\left(\Theta \propto \operatorname{Re}^{-0.23}\right)$ and both $\mathrm{NaCl}$ brine solutions $\left(\Theta \propto \mathrm{Re}^{-0.33} ; \Theta \propto \mathrm{Re}^{-0.38}\right)$. 
Following these considerations, RMF may be successfully applied for enhancement of mixing process for various kinds of liquids. It should be noticed that RMF may induce electromagnetic forces in the mixed liquid due to interactions between the induced electric currents and the applied magnetic field (Mehedintu and Berg, 1997). The application of time-varying magnetic field (e.g. RMF) involves the induction of eddy currents in liquids, which are highly conductive due to additional ions added. These eddy currents create their own magnetic field that in a co-operation with the MF used for the exposure, creates small dynamos mixing the liquid at micro-level. Such microscopic dynamos can enhance the mixing process (Hristov, 2010).

The obtained results may also be analytically described by a unique monotonic function. The plot of data obtained in the current work is presented in Fig. 7a. The calculated values from the proposed relationship (see Fig. 7a) for the mixing process under the action of the RMF and the values obtained from the experimental procedures are graphically compared in Fig. 7b. Fig. $7 \mathrm{~b}$ shows that most results do not exceed the $\pm 15 \%$ maximum error.
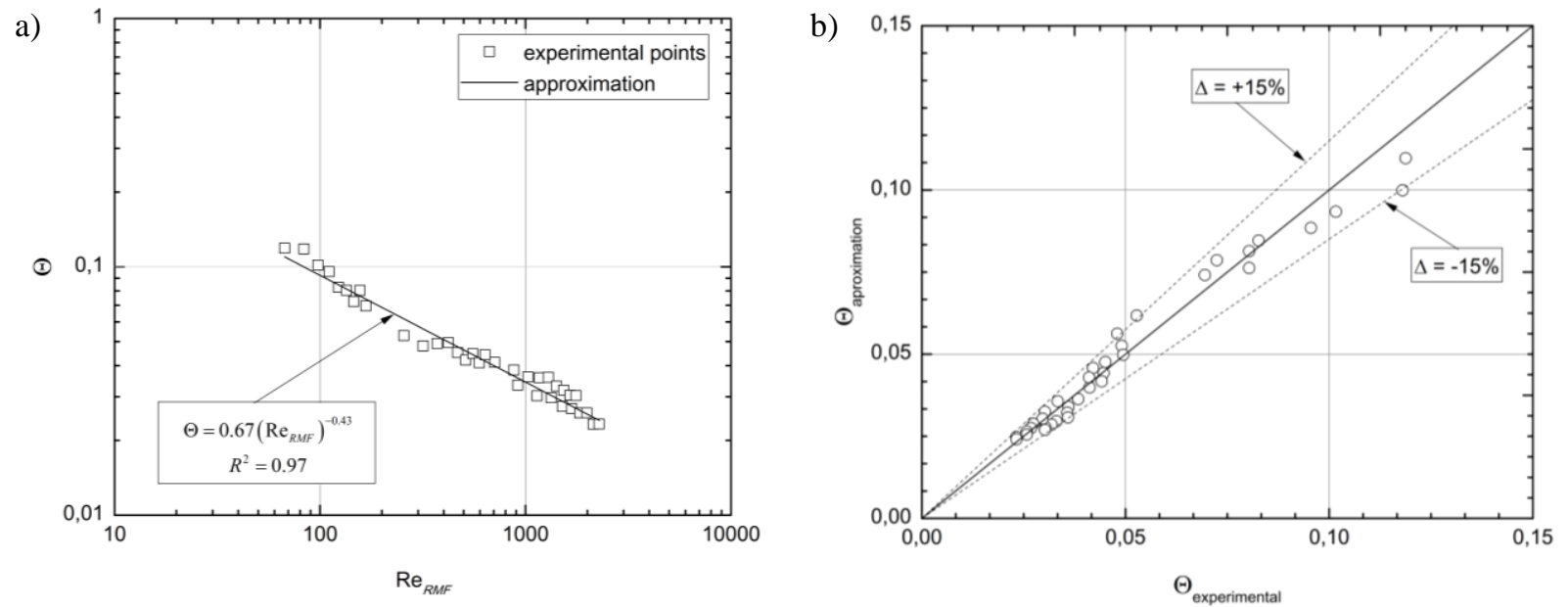

Fig. 7. The dependence of $\Theta=f\left(\operatorname{Re}_{R M F}\right)$ (a) and comparison of the experimental and calculated values or the dimensionless mixing time number $(\mathrm{b})$

It should be noticed that the mixing time can be considered as a criterion for comparison of mixing performance of different studied configurations of mixing devices. Therefore, a comparison between the presented results (see Fig. 7) with experimental findings reported in relevant literature should be done. A comparison of the dimensionless mixing time data from this work and previous studies is given in Fig. 8.

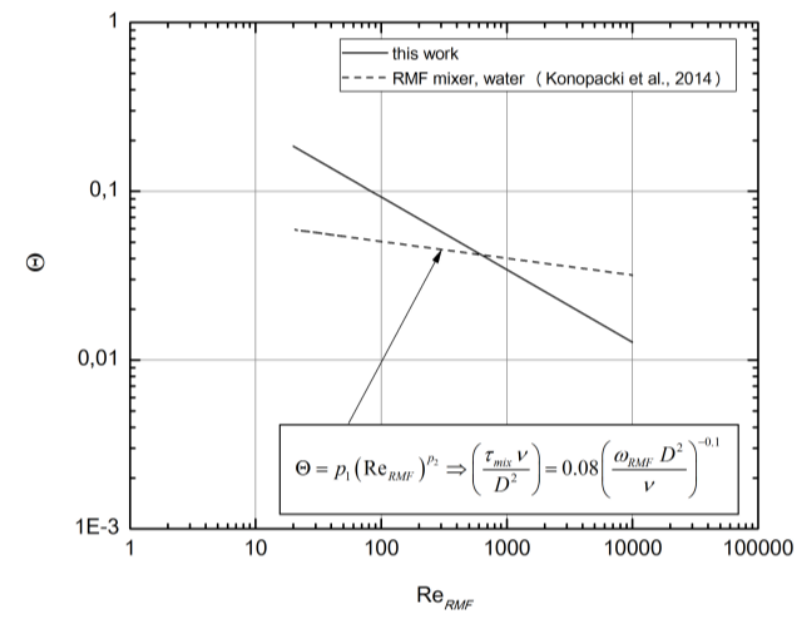

Fig. 8. A comparison of the dimensionless mixing time for systems enhanced by RMF and the RMF mixer (Konopacki et al., 2014) 
Konopacki et al. (2014) studied the effect of RMF and various types of magnetic particles on the mixing time. This study has shown that the mixing time for distilled water (working volume equal to $3.3 \mathrm{dm}^{3}$ ) under the action of the RMF (with the variation of the maximum value of the MF in the range between $23 \mathrm{mT}$ and $34 \mathrm{mT}$ ) decreases with increasing the dimensionless Reynold number. It can be seen that the obtained values of mixing time are consistent with the information from relevant literature.

The values of the dimensionless mixing time for the tested RMF mixer are higher in comparison with the data given in the previously mentioned literature for the dimensionless Reynolds number $\mathrm{Re}_{\mathrm{RMF}}<600$ (Konopacki et al., 2014). A possible explanation for this might be that the applied RMF generator and the working volumes of liquids for the analyzed mixers were different. Manna (1997) proposed that the definition of the mixing time highly depends on the measurement methods, the geometrical configurations of mixers, the system homogeneity and the tool by which the tracer is injected and its location. Therefore, different results of the dimensions mixing time may be explained by the difference between the mixing systems and the use of different methods for mixing time investigations. The current study found that RMF may enhance the mixing process of various types of liquids, e.g.: HS medium, aqueous solutions of brine. As was mentioned above, the magnetic field creates dynamos mixing the liquid at micro-level. These dynamos may be converted into micro-stirrers when the liquid with ions is subjected to RMF. Moreover, micro-stirrers may generate liquid eddies including enhancement of liquid transport properties (Rakoczy, 2013).

\section{CONCLUSIONS}

The analysis of experimental mixing time for the vessel equipped with a RMF generator in the present study leads to the following conclusions:

- The mixing system with the RMF generator may be successfully applied as a mixer for liquids. The experimental data indicated that the influence of RMF on the mixing intensity strongly depends on the physical parameters of liquids.

- The established mathematical correlations describe unitary of the dependence between the mixing time and the modified Reynolds number. The proposed relationship takes into consideration the operational parameters, which define hydrodynamic state and the intensity of the magnetic effects in the tested apparatus.

- The values obtained for the dimensionless mixing time may be used to compare the mixing process performed by various types of mixing systems. It should be noticed that the values of the dimensionless mixing time number for the mixing system with the RMF generator in the region of the lowest Reynolds numbers are much lower than those for STR with the Rushton turbine.

This study was supported by the National Centre for Research and Development in Poland (Grant no. LIDER/011/221/L-5/13/NCBR/2014).

\section{SYMBOLS}

$\begin{array}{ll}B_{\max } & \text { maximum magnetic induction, } \mathrm{kg} \cdot \mathrm{A}^{-1} \cdot \mathrm{s}^{-2} \\ D & \text { diameter of container, } \mathrm{m} \\ f & \text { frequency of electrical current, } \mathrm{s}^{-1} \\ H^{*} & \text { normalized height of a glass container } \\ p H_{0} & \text { initial } \mathrm{pH} \text { values } \\ p H_{\infty} & \text { final } \mathrm{pH} \text { values }\end{array}$




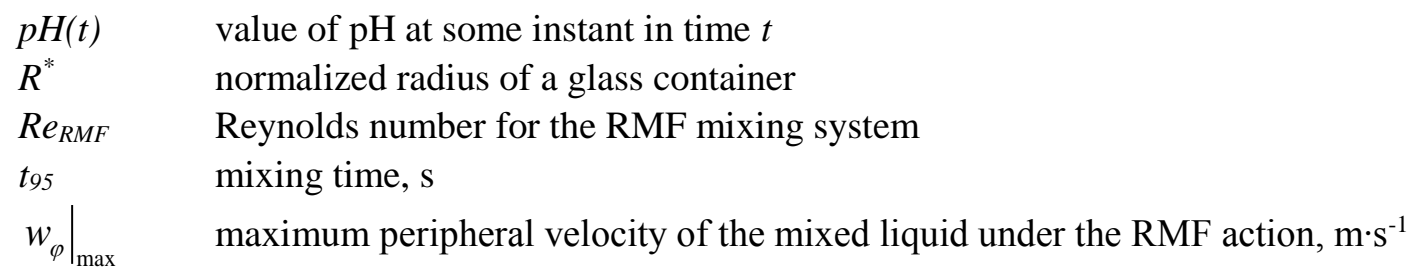

\section{Greek symbols}

$\eta \quad$ dynamic density, $\mathrm{Pa} \cdot \mathrm{s}^{-1}$

$\Theta \quad$ mixing time number

$v \quad$ kinematic viscosity, $\mathrm{m}^{2} \cdot \mathrm{s}^{-1}$

$\rho \quad$ density, $\mathrm{kg} \cdot \mathrm{m}^{-3}$

$\sigma_{e} \quad$ electrical conductivity, $\mathrm{A}^{2} \cdot \mathrm{s}^{3} \cdot \mathrm{kg}^{-1} \cdot \mathrm{m}^{-3}$

$\tau_{\text {mix }} \quad$ mixing time, $\mathrm{s}$

$\omega_{R M F} \quad$ angular velocity of $\mathrm{RMF}, \mathrm{rad} \cdot \mathrm{s}^{-1}$

$\Omega_{R M F} \quad$ angular velocity of liquid under the action of the RMF, $\mathrm{m} \cdot \mathrm{s}^{-1}$

\section{REFERENCES}

Bouaifi M., Roustan M, 2001. Power consumption, mixing time and homogenisation Energy in dual-impeller agitated gas-liquid reactors. Chem. Eng. Process., 40, 87-95. DOI: 10.1016/S0255-2701(00)00128-8.

Bouaifi M., Roustan M., 2001. Power consumption, mixing time and homogenization energy in dual-impeller agitated gas-liquid reactors. Chem. Eng. Process., 40, 87-95. DOI: 10.1016/S0255-2701(00)00128-8.

Cascaval D., Galaction A.-I., Oniscu C., Ungureanu F., 2004. Modeling of mixing in stirred bioreactors 4. Mixing time for aerated bacteria, yeasts and fungus broths. Chem. Indust., 58, 128-137. DOI: 10.2298/HEMIND0403128C.

Ciechańska D., Struszczyk H., Gruzińska K., 1998. Modification of bacterial cellulose. Fibres Text. East. Eur., 4(23), 61-65.

Dahlberg E., 1972. On the action of a rotating magnetic field on a conducting liquid. Aktiebolaget Atomenergi, Studsvik, Nyköping, Sweden.

Fijałkowski K., Żywicka A., Drozd R., Niemczyk A., Junka A.F, Peitler D., Kordas M., Konopacki M., Szymczyk P., El Fray M., Rakoczy R., 2015. Modification of bacterial cellulose through exposure to the rotating magnetic field. Carbohyd. Polym., 133, 52-60. DOI: 10.1016/j.carbpol.2015.07.011.

Gaafar E.-S.A., Hanafy M.S., Tohamy E.Y., Ibrahim M.H., 2008. The effect of electromagnetic field on protein molecular structure of E. coli and its pathogenesis. Rom. J. Biophys., 18, 145-169.

Hadjiev D., Sabiri N.E., Zanati A., 2006. Mixing time in bioreactors under aerated conditions. Biochem. Eng. J., 27, 323-330. DOI: 10.1016/j.bej.2005.08.009.

Harnby N., Edwards M.F., Nienow A.W. Mixing in the process industries. Butterworth-Heinemann, Bosotn, 2000 .

Hiraoka S., Kato Y., Tada Y., Ozaki N., Murkami Y., Lee Y.S., 2001. Power consumption and mixing time in an agitated vessel with double impeller. Chem. Eng. Res. Des., 79, 805-810. DOI: 10.1205/02638760152721613.

Hristov J., 2010. Magnetic field assisted fluidization-a unified approach. Part 8. Mass transfer: Magnetically assisted bioprocess. Rev. Chem. Eng., 26, 55-128. DOI: 10.1515/REVCE.2010.006.

Jaworski Z., Bujalski W., Otomo N., Nienow A.W., 2000. CFD study of homogenization with dual Rushton turbines - Comparison with experimental results. Part I: Initial studies. Chem. Eng. Res. Des., 78, 327-333. DOI: $10.1205 / 026387600527437$.

King R.L., Hiller R.A., Tatterson G.B., 1988. Power consumption in a mixer. AIChE J., 34, 506-509. DOI: 10.1002/aic.690340320.

Konopacki M., Frąckowiak A., Tabero P., Fijałkowski K., Rakoczy R., 2014. Studies of a mixing process by using the various types of magnetic particles as micro-stirrers. Technical Transactions. Chemistry, 24, 45-54.

Kordas M., Story G., Konopacki M., Rakoczy R., 2013. Study of mixing time in a liquid vessel with rotating and reciprocating agitator. Ind. Eng. Chem. Res., 52, 13818-13828. DOI: 10.1021/ie303086r. 
Kushalkar K.B., Pangarkar V.G., 1994. Particle - liquid mass transfer in a bubble column with a draft tube. Chem. Eng. Sci., 49, 139-144. DOI:10.1016/0009-2509(94)85041-0.

Magelli F., Montante G., Pinelli D., Paglianti A., 2013. Mixing time in high aspect ratio vessels stirred with multiple impellers. Chem. Eng. Sci., 101, 712-720. DOI: 10.1016/j.ces.2013.07.022.

Manjula P., Kalaichelvi P., Dheenathayalan K., 2010. Development of mixing time correlation for a double jet mixer. J. Chem. Technol. Biot., 85, 115-120. DOI: 10.1002/jctb.2274.

Manna L., 1997. Comparison between psychical and chemical methods for the measurement of mixing time. Chem. Eng. J., 67, 167-173. DOI: 10.1016/S1385-8947(97)00059-4.

Masiuk S., Rakoczy R., 2007. Power consumption, mixing time, heat and mass transfer measurements for liquid vessels that are mixed using reciprocating multiplates agitators. Chem. Eng. Process., 46, 89-98. DOI: 10.1016/j.cep.2006.05.002.

Masiuk S., Rakoczy R., Kordas M., 2008. Comparison density of maximal energy for mixing process using the same agitator in rotational and reciprocating movements. Chem. Eng. Process., 47, 1252-1260. DOI: 10.1016/j.cep.2007.04.004.

Mehedintu M., Berg H., 1997. Proliferation response of yeast Saccharomyces cerevisiae on electromagnetic filed parameters. Bioelectroch. Bioener., 43, 67-70. DOI: 10.1016/S0302-4598(96)05184-7.

Moffatt H.K., 1965. On fluid flow induced by rotating magnetic field. J. Fluid. Mech., 22(3), 521-528. DOI: $10.1017 /$ S0022112065000940.

Moffatt H.K., 1991. Electromagnetic stirring. Phys. Fluids A, 3(5), 1336-1343. DOI: 10.1063/1.858062.

Oniscu C., Galaction A-I., Cascaval D., Ungureanu F., 2002. Modeling of mixing in stirred bioreactors: 2. Mixing time for non-aerated broths. Biochem. Eng. J., 12, 61-69. DOI: 10.1016/S1369-703X(02)00042-6.

Rakoczy R., 2013. Mixing energy investigations in a liquid vessel that is mixed by using a rotating magnetic field. Chem. Eng. Process., 66, 1-11. DOI: 10.1016/j.cep.2013.01.012.

Rivera Ch., Foucault S., Heniche M., Espinosa-Solares T., Tanguy P. A., 2006. Mixing analysis in a coaxial mixer. Chem. Eng. Sci., 61, 2895-2907. DOI: 10.1016/j.ces.2005.11.045.

Story G., Kordas M., Rakoczy R., 2016. Correlations for mixing energy in processes using Rushton turbine mixer. Chem. Pap., 70, 747-756. DOI: 10.1515/chempap-2016-0008.

Stręk F. Mieszanie i mieszalniki. WNT, Warszawa, 1981.

Szoplik J., Karcz J., 2008. Mixing time of a non-Newtonian liquid in an unbaffled agitated vessel with an eccentric propeller. Chem. Pap., 62, 70-77. DOI: 10.2478/s11696-007-0081-9.

Woziwodzki Sz., Broniarz-Press L., Ochowiak M., 2010. Effect of Eccentricity on transitional mixing in vessel equipped with turbine impellers. Chem. Eng. Res Des., 88, 1607-1614. DOI: 10.1016/j.cherd.2010.04.007.

Zadghaffari R., Moghaddas J. S., Revstedt J., 2007. A mixing study in a double-Rushton stirred tank. Comput. Chem. Eng., 33, 1240-1246. DOI: 10.1016/j.comp-chemeng.2009.01.017.

Zlotarnik M. Stirring: Theory and practice. Wiley-VCH, Weinheim, 2001.

Received 28 September 2016

Received in revised form 16 November 2017

Accepted 18 November 2017 